\title{
Research on Long-Distance Air Combat Attach Placeholder Based on SOS Algorithm
}

\author{
Zhihong Wang ${ }^{\mathrm{a}}$, Xianzhi Wei, Jianrui $\mathrm{Xu}{ }^{\mathrm{b}}$, Baofeng Wang
}

Engineering College of Aeronautics and Astronautics, Air Force Engineering University, Xi'an 710038, China

awangzhihong7285829@163.com, bxjrcr@163.com

Keywords: long-distance air combat; tactics decision; SOS algorithm; placeholder.

\begin{abstract}
Based on long-distance air combat tactics decision and against the long-distance autonomous attach placeholder problem of UCAV, the paper proposes a tactics maneuvering decision method. It constructs a threat evasion objection function and rapid target approach attack objective function, selects corresponding objective function based on tactics decision result and adopts modified SOS algorithm to optimize the maneuvering decision control variable.
\end{abstract}

\section{Introduction}

Under the promotion of new revolution in military affairs, the development of unmanned aerial vehicle (UAV) is in the ascendant. Especially, with the in-depth research on computer technology and artificial intelligence technology (AIT), various UAVs with extraordinary performance, advanced technology and wide purpose spring up like mushrooms after rain. As an important direction of UAV [1], Unmanned Combat Aerial Vehicle (UCAV) has great development potential.

Compared with genetic algorithm (GA), the intelligent algorithm, such as Symbiotic Organisms Search (SOS) algorithm ${ }^{[2]}$, particle swarm optimization (PSO) ${ }^{[3]}$ and artificial bee colony algorithm $(\mathrm{ABC})^{[4]}$ are operated simple, have fewer control parameters, good stability and strong optimization ability. Once proposed, it was concerned and studied by many scholars. The paper utilizes SOS algorithm for optimization of UCAV maneuvering decision control parameter so as to meet the decision requirement and optimize the decision effect.

\section{SOS algorithm}

Standard SOS algorithm has three operation stages: mutualism, commensalism and parasitism. The basic thought is to simulate the survival relationship among creatures and produce intelligent guidance optimization searching of swarm through cooperation and competition among individuals in the population. The main procedures of the algorithm are as below:

\subsection{Initialization.}

The algorithm should be firstly initialized as per the following formula:

$\boldsymbol{X}_{i}=\boldsymbol{L} \boldsymbol{b}+\operatorname{rand}(0,1) \cdot(\boldsymbol{U} \boldsymbol{b}-\boldsymbol{L} \boldsymbol{b})$

$N P$ initial solutions are generated at random. Hereinto, $\boldsymbol{X}_{i}$ represents $i$ th creature in the ecological system, the dimension is $D, \boldsymbol{U} \boldsymbol{b} 、 \boldsymbol{L} \boldsymbol{b}$ are the upper limit and lower limit of searching space and $\operatorname{rand}(0,1)$ is the random number in the range of $(0,1)$.

\subsection{Mutualism searching stage.}

Select a "creature" from the popular at random, $\boldsymbol{X}_{j}$ and $\boldsymbol{X}_{i}$ are interacted, which makes them learn from optimal solution. $\boldsymbol{X}_{i}$ and $\boldsymbol{X}_{j}$ generate new $\boldsymbol{X}_{\text {inew }}$ and $\boldsymbol{X}_{\text {jnew }}$ according to the following formula. 


$$
\begin{aligned}
& \boldsymbol{X}_{\text {inew }}=\boldsymbol{X}_{i}+\varphi \cdot\left(\boldsymbol{X}_{\text {best }}-\text { Mutual_Vector } \cdot B F_{1}\right) \\
& \boldsymbol{X}_{\text {jnew }}=\boldsymbol{X}_{j}+\varphi \cdot\left(\boldsymbol{X}_{\text {best }}-\text { Mutual_Vector } \cdot B F_{2}\right) \\
& \text { Mutual_Vector }=\frac{\boldsymbol{X}_{i}+\boldsymbol{X}_{j}}{2}
\end{aligned}
$$

Hereinto, $i, j \in\{1,2, \ldots N P\}(i \neq j)$, zoom factor $\varphi$ is a random number in the range of $[0,1]$. $\boldsymbol{X}_{\text {best }}$ is optimal individual at present, $B F_{1} 、 B F_{2}$ express gain factor, which is 1 or 2 at random.

\subsection{Commensalism searching stage.}

Select a "creature" from the popular at random, $\boldsymbol{X}_{j}$ and $\boldsymbol{X}_{i}$ are interacted $(i \neq j), \boldsymbol{X}_{\text {inew }}$ through the following formula.

$$
\boldsymbol{X}_{\text {inew }}=\boldsymbol{X}_{i}+\psi \cdot\left(\boldsymbol{X}_{\text {best }}-\boldsymbol{X}_{j}\right)
$$

Hereinto, zoom factor $\psi$ is a random number in the range of $[-1,1]$.

\subsection{Population updating stage.}

Through selecting parameters of some dimension from $\boldsymbol{X}_{i}$ for random revision, a variation individual can be got, called "parasitism vector", recorded as $\boldsymbol{X}_{p v}$; select individual $\boldsymbol{X}_{j}(j \neq i)$ as the "host" of $\boldsymbol{X}_{p v}$ and make comparison. If $\boldsymbol{X}_{p v}$ is superior to $\boldsymbol{X}_{j}$, "creature" $\boldsymbol{X}_{j}$ will be killed and substituted by $\boldsymbol{X}_{p v}$; on the contrary, $\boldsymbol{X}_{j}$ is immune to $X_{p v}$, it continues surviving and remains in the population.

\section{Three DOF model of UCAV}

\subsection{Difference equation of state variable updating.}

Based on UCAV particle kinematics and kinetic equation, the state variable of UCAV can be expressed as $\boldsymbol{X}=(x, y, z, \gamma, \psi, V)$. Hereinto, the various express coordinate $x$ and $y$, height, track angle, course angle and velocity and control variable is expressed with $\boldsymbol{u}=\left[n_{x}, n_{z}, \boldsymbol{\phi}\right]$.

At the time $t_{k}$, the current state of UCAV is decided by the state parameter of the last moment and maneuvering decision.

$$
\boldsymbol{X}_{k+1}=\boldsymbol{X}_{k}+f\left(\boldsymbol{X}_{k}, \boldsymbol{u}_{k}\right) \Delta t \quad(k=0,1, \ldots, N-1)
$$

Hereinto,

$$
f\left(\boldsymbol{X}_{k}, \boldsymbol{u}_{k}\right)=\left\{\begin{array}{c}
V_{k} \cos \gamma_{k} \cos \psi_{k} \\
V_{k} \cos \gamma_{k} \sin \psi_{k} \\
V_{k} \sin \gamma_{k} \\
g\left(n_{x . k}-\sin \gamma_{k}\right) \\
\frac{g}{V_{k}}\left(n_{z . k} \cos \phi_{k}-\cos \gamma_{k}\right) \\
\frac{g n_{z . k} \sin \phi_{k}}{V_{k} \cos \gamma_{k}}
\end{array}\right.
$$

\subsection{Relative geometric relationship of air combat.}

The azran between UCAV and target is expressed as $\boldsymbol{C}=\left(\varphi_{u}, q_{u}, R\right)$. Hereinto, $\varphi_{u}$ is the azimuth angle of UCAV, $q_{u}$ is the target entrance angle, $R$ is the distance between two aircrafts. $\boldsymbol{C}=\left(\varphi_{u}, q_{u}, R\right)$ describes the relative position relationship between UCAV and target, as shown in Fig. 1. $\varphi_{u}, q_{u}$ and $R$ can be calculated through Formula (7)-(9). 


$$
\begin{gathered}
\overrightarrow{\boldsymbol{V}_{\mathrm{u}}}=\left[\begin{array}{l}
V_{\mathrm{u}} \cos \gamma_{\mathrm{u}} \cos \psi_{\mathrm{u}} \\
V_{\mathrm{u}} \cos \gamma_{\mathrm{u}} \sin \psi_{\mathrm{u}} \\
V_{\mathrm{u}} \sin \gamma_{\mathrm{u}}
\end{array}\right] \\
\varphi_{u}=\arccos \left(\frac{\overrightarrow{\boldsymbol{R}} \cdot \overrightarrow{\boldsymbol{V}}_{u}}{\|\overrightarrow{\boldsymbol{R}}\| \cdot\left\|\overrightarrow{\boldsymbol{V}}_{u}\right\|}\right) \\
q_{u}=\arccos \left(\frac{\overrightarrow{\boldsymbol{R}} \cdot \overrightarrow{\boldsymbol{V}}_{t}}{\|\overrightarrow{\boldsymbol{R}}\| \cdot \| \overrightarrow{\boldsymbol{V}_{t} \|}}\right) \\
\overrightarrow{\boldsymbol{R}}=\left[x_{t}-x_{u}, \quad y_{t}-y_{u}, \quad z_{t}-z_{u}\right]
\end{gathered}
$$

In the formula, $\varphi \in[0, \pi], q \in[0, \pi]$, the subscript $u, t$ represent UCAV and target.

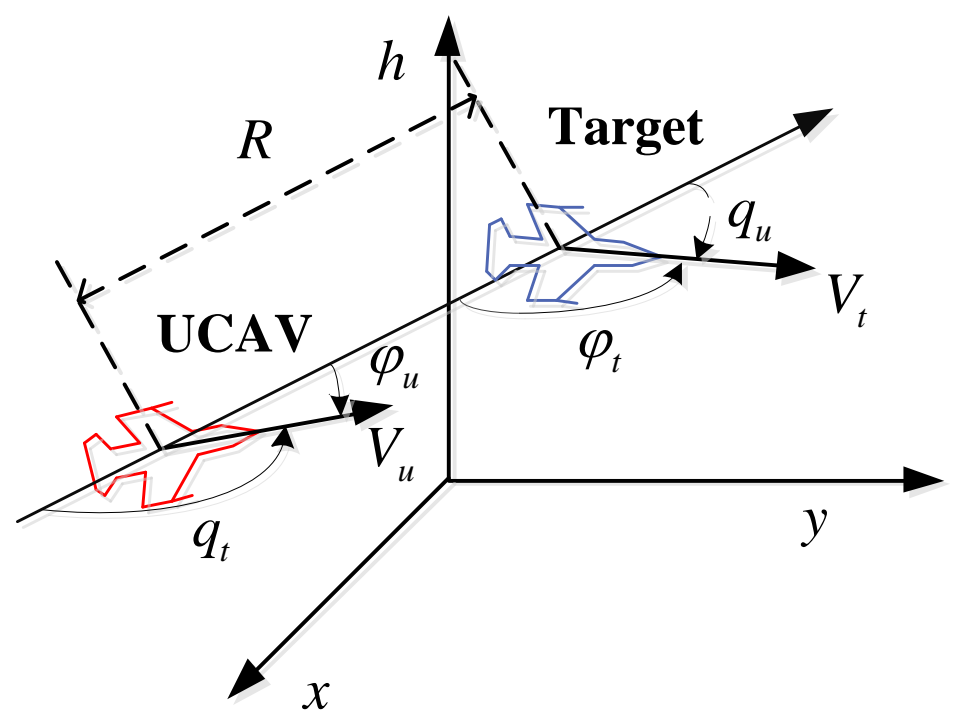

Fig 1. Azran sketch

\section{Influencing factor analysis of maneuvering placeholder}

\subsection{Air war situation analysis.}

Based on the relative azimuth angle between UCAV and target and combined with maximum detection range of radar and attack distance of missile, the threatening area and advantageous area are constructed.

Advantageous area:

$$
\text { zone }_{p r i}=\left\{s \mid \varphi_{u} \leq \psi_{u}, \varphi_{t} \geq \psi_{t}, L \leq R_{U} \cup L \in\left[R_{U \text { min }}, R_{U \text { max }}\right]\right\}
$$

In the formula, $\varphi_{u}, \psi_{u}$ and $R_{D}$ are azimuth angle of UCAV, maximum searching angle of radar and maximum detection distance; $\varphi_{t}, \psi_{t}$ and $R_{U}$ are target azimuth angle and maximum searching angle of radar; $L$ is relative distance between two aircrafts; $R_{g \text { min }}$ and $R_{g \text { max }}$ are minimum and maximum attack distance of UCAV missile.

Threatening area:

$$
\text { zone }_{e x}=\left\{s \mid \varphi_{t} \leq \psi_{t}, L \leq R_{T} \cup L \in\left[R_{T \text { min }}, R_{T \max }\right]\right\}
$$

In the formula, $R_{T \text { min }}$ and $R_{T \text { max }}$ express minimum and maximum attack distances of target missile, $R_{T}$ expresses the maximum detection distance of target radar. 


\subsection{Design of long-distance attack placeholder objective function.}

\subsubsection{Evade threat objective function}

Prior to missile attack, it needs radar to lock. The missile threatening area is usually included in the radar detection area. So the evasion to target should adopt the evasion to radar detection area.

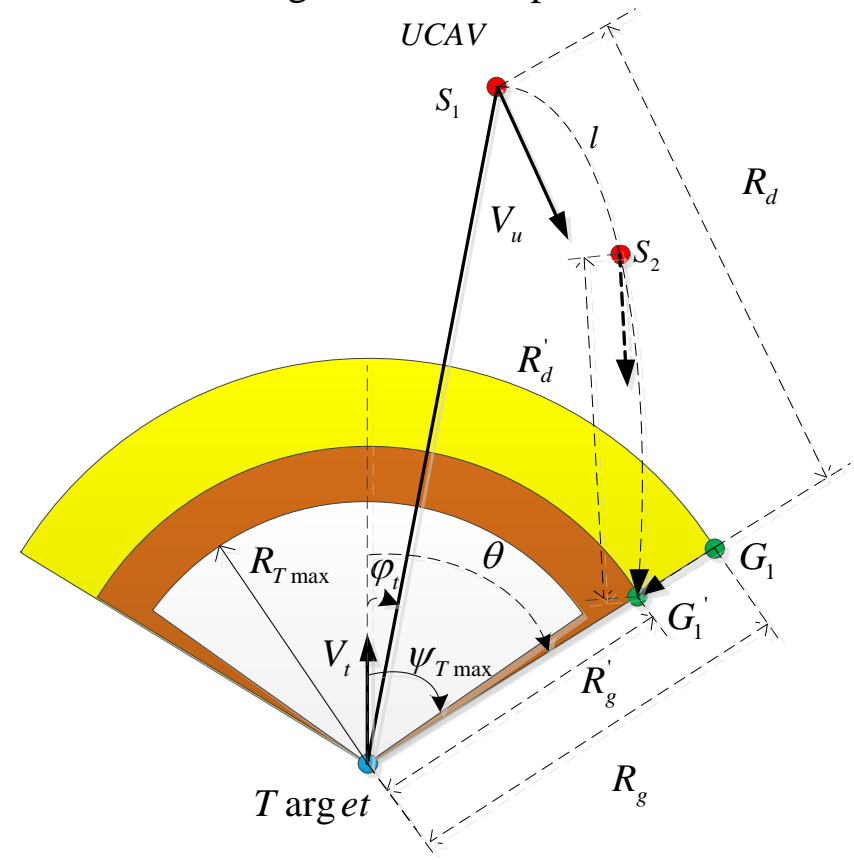

Fig. 2 Plan sketch of dynamic leading point theory

Hereinto, red dot presents UCAV, blue dot presents target, green dot $G_{1}$ is leading point, $S_{1} 、 S_{2}$ are the positions of UCAV at different moment, and curve $l$ represents the final track of UCAV.

The basic thought of dynamic leading theory is: firstly increase the maximum searching angle and detection distance of target radar properly, get a sector area with the angle $\theta$ and radius $R_{g}$. Then, take the external boundary point of the sector area as the leading point. Hereinto, radius $R_{g}$ decreases with the increasing of target azimuth angle $\varphi_{t}$. When the target azimuth angle is small, it represents the target nose points at UCAV, going against UCAV. Therefore, UCAV should move toward outside area to increase the target azimuth angle. The bigger the radius $R_{g}$ is, the more the leading point is outside of target in favor of UCAV to evade threat. With the increasing of target azimuth angle, the treat to UCAV becomes small, the radius $R_{g}$ of leading point becomes small gradually and leads UCAV to approach to target so as to realize side around target approach purpose. The flight track sketch is shown as $l$ in Fig. 2 . The radius $R_{g}$ change of leading point with the change of target azimuth angle can be expressed with Formula (12).

$$
R_{g}=R_{T \max }\left(2-e^{-\frac{\varphi_{t}}{\theta}}\right)
$$

Hereinto, $\theta>\varphi_{t}$. According to Formula (12), it can be known that radius of leading point is always greater than maximum detection distance of target radar, namely $R_{g} \geq R_{T \max }$. Therefore, UCAV is located outside the target detection area in the overall process. To make UCAV have a certain height advantage after evading threat, the relative target of leading point should have a certain height difference $\Delta h_{g}$.

It needs to approach leading point to evade threat during attack placeholder process. Therefore, the objective function of threat evasion is constructed as: 


$$
\mu\left(R_{d}\right)=\left\{\begin{array}{cl}
\frac{1}{1+R_{d}} & 0 \leq \varphi_{t}<\psi_{T \max } \\
1 & \psi_{T \max } \leq \varphi_{t}
\end{array}\right.
$$

In the formula, $R_{d}$ is relative distance between UCAV and leading point.

\subsubsection{Rapid target approach attack objective function}

After UCAV evades the detection threat of target, it should complete placeholder as much as possible so as to form missile launching condition, which has requirement in azimuth angle, relative distance, height and velocity of UCAV. Therefore, to meet the placeholder requirement, evaluation functions should be firstly established for these four parameters

(1)Angle evaluation function

$$
\mu\left(\varphi_{u}\right)=e^{-\frac{\varphi_{u}}{\pi}}
$$

(2) Distance evaluation function

$$
\mu(R)= \begin{cases}e^{-\left(1-\frac{R_{*}}{R}\right)} & R>R_{*} \\ e^{-\left(1-\frac{R}{R_{*}}\right)} & R \leq R_{*}\end{cases}
$$

In Formula (15), $R$ is relative straight-line distance between UCAV and target; $R_{*}$ is the expected attack distance. The expression is:

$$
R_{*}=R_{k \min }+\frac{R_{k \max }-R_{k \min }}{2}
$$

(3) Height evaluation function

$$
\mu(H)= \begin{cases}e^{-\left(1-\frac{\Delta h_{*}}{\Delta h}\right)} & \Delta h>\Delta h_{*} \\ e^{-\left(1-\frac{\Delta h}{\Delta h_{*}}\right)} & \Delta h \leq \Delta h_{*}\end{cases}
$$

In the formula, $\Delta h=h_{u}-h_{t}$ is the height difference between UCAV and target.

(4) Velocity evaluation function

$$
\mu(V)= \begin{cases}e^{-\left(1-\frac{V_{*}}{V}\right)} & V>V_{*} \\ e^{-\left(1-\frac{V}{V_{*}}\right)} & V \leq V_{*}\end{cases}
$$

Hereinto, $V_{*}$ is the expected velocity

$$
V_{*}= \begin{cases}V_{t}+\left(V_{u \max }-V_{t}\right)\left(1-e^{-\left(\frac{R_{t}}{R}\right)^{2}}\right), & R \geq R_{*} \\ V_{t}+\left(V_{u \min }-V_{t}\right)\left(1-e^{-\left(\frac{R}{R_{s}}\right)^{2}}\right), & R<R_{*}\end{cases}
$$

To sum up, rapid target approach attach indicator is constructed as

$$
\mu_{\text {attack }}=\omega_{1} \mu\left(\varphi_{u}\right)+\omega_{2} \mu(R)+\omega_{3} \mu(H)+\omega_{4} \mu(V)
$$

\subsection{Optimization decision based on SOS algorithm.}

For the control variable optimization of intelligent algorithm, the thought is to take control vector as the solution vector of algorithm. Then, take the maneuvering decision objective function under the corresponding tactics as the fitness function for optimization and constant iteration until find the optimal solution. The optimization method is substituting control variable into particle model and get all possible positions under UCAV. Through evaluation function $J$, it solves the situation of enemy and ourselves at each position so as to select the control variable with optimal situation.

Specific procedures are as below: 
Step 1: according to the current combat status, make tactics decision and select objective function $J$ as the fitness function.

Step 2: according to control variable, determine the value range of problem parameter and each dimension of parameter, initialize $N P$ colonial individuals and set the maximum number of iteration as $G_{\max }$.

Step 3: Substitute each solution into algorithm for optimization and constant iteration until reach maximum number of iteration.

Step 4: after completing all the iterations, output the found global optimal solution, namely the optimal control variable.

The tactics maneuvering decision process based on SOS algorithm is shown as Fig. 3.

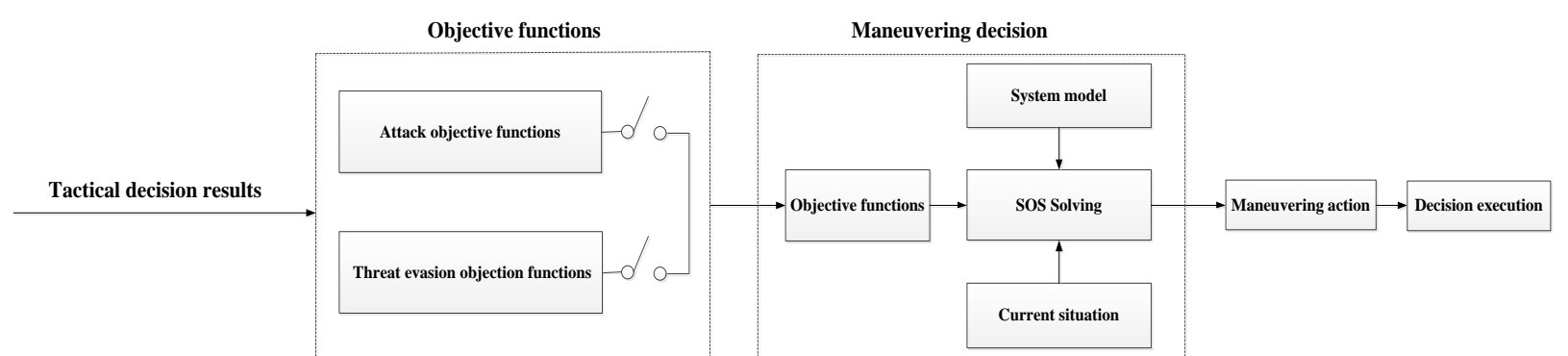

Fig. 3 UCAV long-distance side around attack placeholder tactics maneuvering decision process

\section{Simulation verification and analysis.}

The chapter mainly studies attach placeholder maneuvering decision on long-distance attack condition without considering the correlation. Therefore, we assume the weapon performance and radar performance of both parties at the same level and both parties are possible to win.

Public parameters are set as:

Flight constraint: velocity $[90,350] \mathrm{m} / \mathrm{s}$ and flight height ; controlled variable constraint: tangential overload $n_{x} \in[-1.5,1.5]$, normal overload $n_{z} \in[0,9]$, roll angle $\phi \in[0,2 \pi]$; the maximum horizontal angle of radar $\psi_{\max }=45^{\circ}$, the detection distance for the target with RCS of $3 \mathrm{~m}^{2}$ is $50 \mathrm{~km}$; the farthest effective range of empty missile is $45 \mathrm{~km}$, the maximum unescapably distance is $R_{k \max }=40 \mathrm{~km}$, the minimum unescapably distance is $R_{k \min }=30 \mathrm{~km}$, the maximum off-axial angle of missile launching is $45^{\circ}$, and $\theta=50^{\circ}$; expected height difference is set as $\Delta h_{*}=1000$, the positive height difference of leading point is $\Delta h_{g}=1000$, the expected height difference is $\sigma_{h}=300$; the weight of rapid target approach attack objective function is set as $\omega_{1}=0.3, \omega_{2}=0.3, \omega_{3}=0.2, \omega_{4}=0.2$; decision step size is set as $1 \mathrm{~s}$ and total simulated step size is 300 .

Algorithm parameters are set as: algorithm searching individual is 20, and number of iteration is 20; simulation platform is Matlab R2013a, basic frequency of processor is $3.00 \mathrm{GHz}$ and memory is 3.15GB. When the target is located outside UCAV detection area, the target position information will be transmitted to UCAV by the ground information support platform.

Instance: the target heads on a straight line and is locked by UCAV radar then veers to escape.

Initial position of UCAV is $(100000 \mathrm{~m},-10000 \mathrm{~m}$ and $6000 \mathrm{~m})$; initial speed is $200 \mathrm{~m} / \mathrm{s}$, tilted angle of track is $0^{\circ}$, deflection angle of track is $180^{\circ}$ and initial position of target is $(0 \mathrm{~m}, 0 \mathrm{~m}, 7000 \mathrm{~m})$; Initial velocity is $200 \mathrm{~m} / \mathrm{s}$, tilted angle of track is $0^{\circ}$, and deflection angle of track is $0^{\circ}$. When the relative distance is set to be smaller than maximum attack distance of UCAV, the target is locked.

According to the set initial situation parameter, select the side around tack as the tactics under the current situation. The tactics firstly requires invading the threat from target, then approaching the target and attacking quickly. Suppose the maneuvering status before the target detects UCA is invariant and veers to escape after it is locked by UCAV radar. The simulation result is as below: 


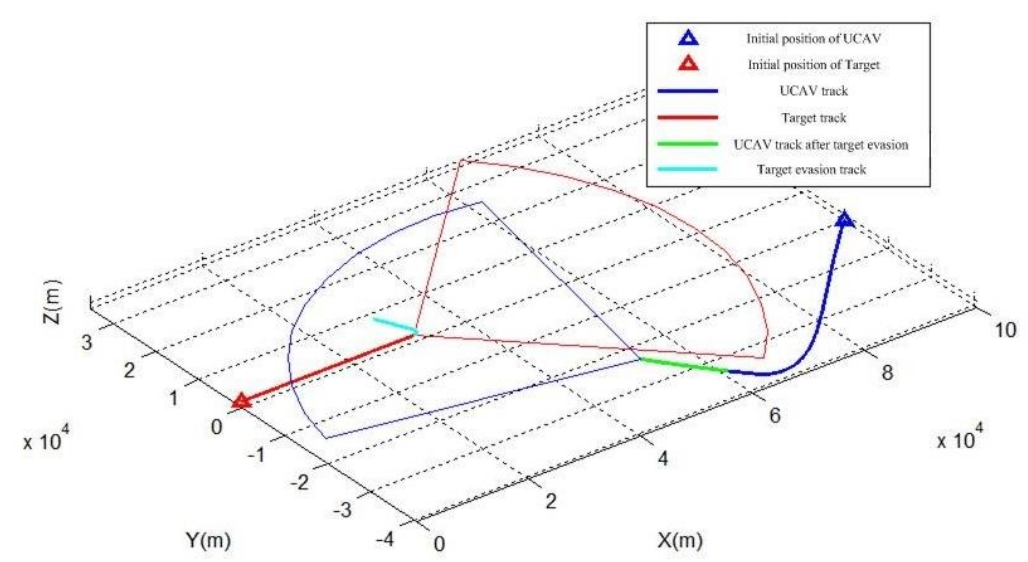

Fig. 4 UCAV long-distance side around attack placeholder track

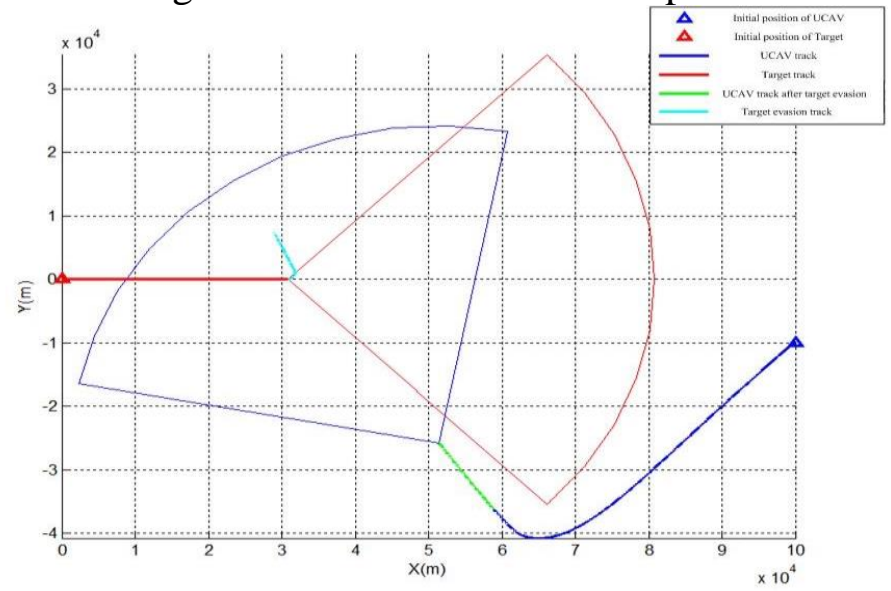

Fig. 5 Top view of UCAV long-distance side around attack placeholder track

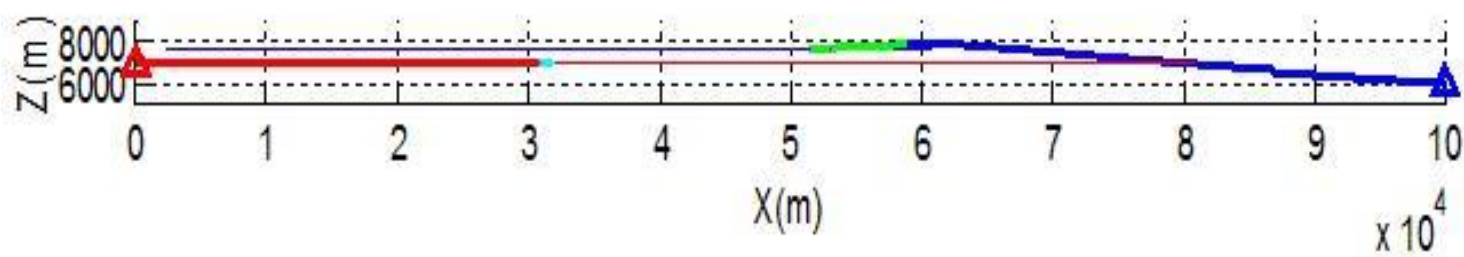

Fig. 6 Side view of UCAV long-distance side around attack placeholder track

Fig. 4 to 6 presents 3D view, top view and side view of UCAV long-distance autonomous attack placeholder maneuvering decision track under the head-on situation. The step size of simulation from starting to ending is $185 \mathrm{~s}$, decision duration is $72.9 \mathrm{~s}$, and average decision duration of each step is $0.39 s$, which meeting the real time requirement. After the simulation starts, according to the tactics requirement, when UCAV approaches the target, it evades the region at the same time. It realizes threat evasion at $144 \mathrm{~s}$ and then turns to rapid target approach attack. UCAV locks target at $155 \mathrm{~s}$. When the target observes that it is locked, it will turn to escape and UCAV completes attack placeholder at $185 s$. then, the simulation comes to an end. Red section area expresses the threat area of target. According to Fig. 5, it can be known that when UCAV is observed, it doesn't enter the threat area of target, so it indicates the target approach strategy is reasonable and effective. According to Fig. 6, it can be known that, at the end of placeholder, UCAV gains a certain height advantage for target and meet the height requirement. Blue sector area expresses threat area of UCAV. The target starts entering radar detection area of UCAV, then attack scope and finally UCAV completes placeholder task. In this way, side around attack tactics is successful. 


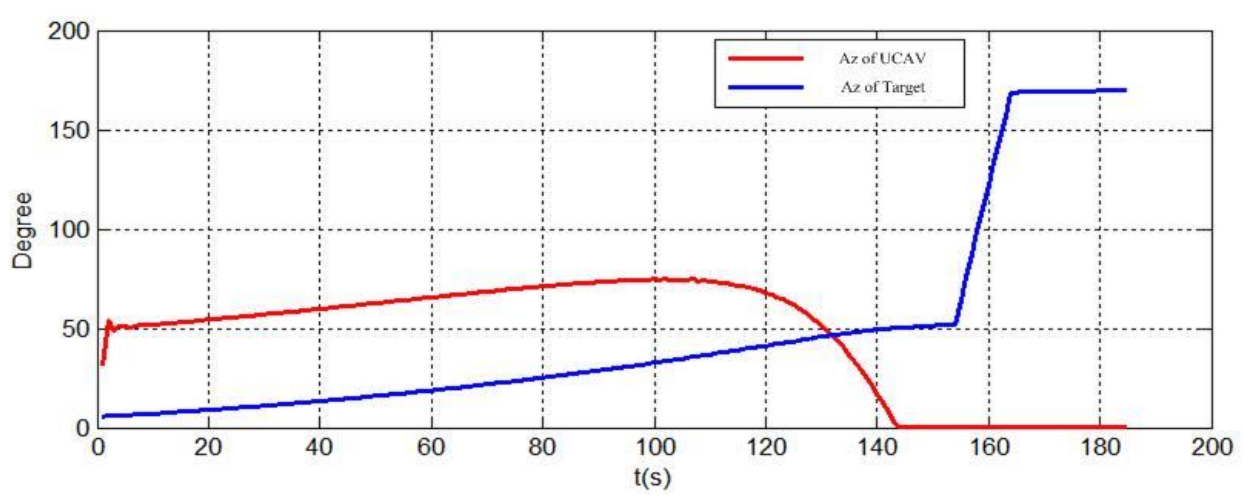

Fig. 7 Related angle changing curve

Fig. 7 is azimuth angle changing curve of UCAV and target during attach placeholder process. The two aircrafts head on at the initial moment. UCAV adopts side around attack tactics and moves to outside of the target to increase the target azimuth angle, as well as making its own azimuth angle change dramatically without heading on the target. After 110s, the dynamic leading point approaches to target, and UCAV azimuth angle component decreases. However, it is still at threat evasion stage. When it comes to $142 \mathrm{~s}$, target azimuth angle is larger than the maximum detection angle of radar, UCAV turns to rapid target approach attach stage and velocity direction points at target so that the azimuth angle changes dramatically. When it comes to 155s, UCAV locks the target; the target turns to escape and makes the azimuth angle increase sharply; when the simulation comes to an end, UCAV azimuth angle $\varphi_{u}=0$, meeting the placeholder requirement.

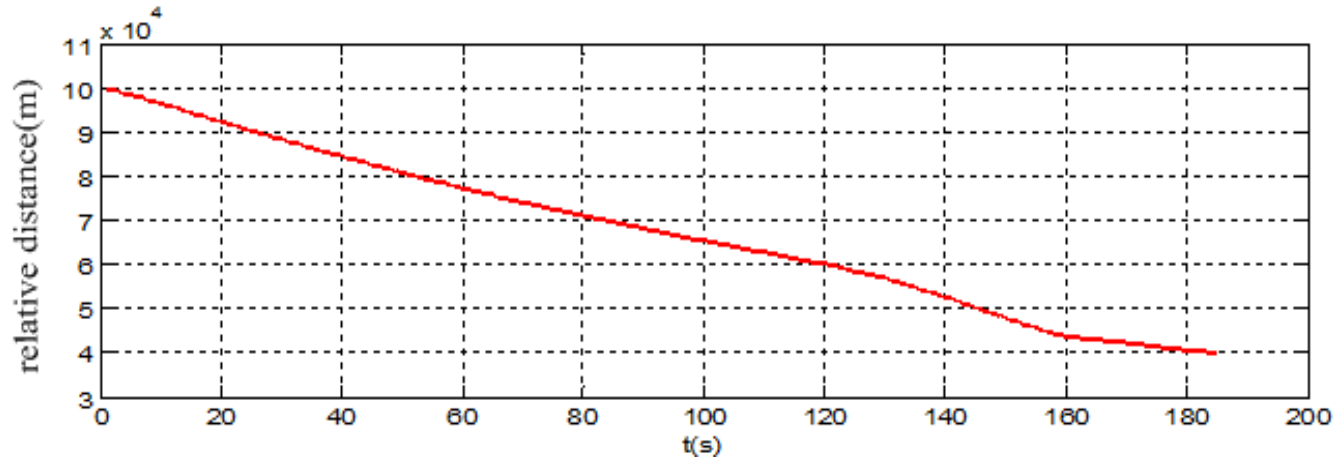

Fig. 8 Relative distance changing curve

Fig. 8 is the relative distance changing curve between UCAV and target. According to Fig. 8, it can be seen that before $155 \mathrm{~s}$, two aircrafts are in opposite-direction movement state and the distance changes rapidly. After 155s, the target escapes, and UCAV chases after it. The distance is reduced constantly, but the change trend is slow. When the simulation is completed, relative distance meets the distance constraint condition at the end of decision.

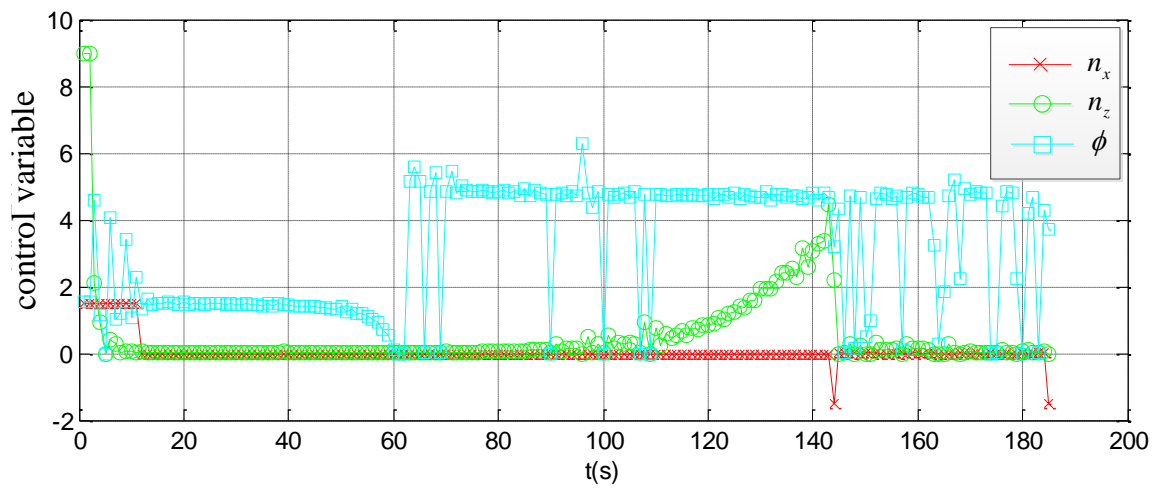

Fig. 9 Controlled variable changing curve

Fig. 9 is changing curve of UCA controlled variable. From Fig. 9, it can be seen that various controlled variables are within the constraint condition scope, which indicates that application algorithm is feasible for optimizing the controlled variable of maneuvering decision. 


\section{Summary}

The conversion between situation and tactics task during the long-distance autonomous attack placeholder cannot use a uniform objective function to constrain the maneuvering decision. Therefore, the paper proposes a maneuvering decision method based on tactics. Against task demand at different stages, the paper constructs threat evasion objective function and rapid target approach attack objective function and UCAV shifts the corresponding objective functions automatically for decision making according to tactics decision result. The paper adopts modified optimization algorithm to solve the controlled variable of decision. The simulation result indicates that the designed objective function is reasonable and meets the tactics requirement under different situations and the proposed tactics maneuvering decision method can implement target threat evasion and target attack autonomously in the attack placeholder process.

\section{References}

[1]. Guo Guangmin,Luo Qin,Zhao Zhiquan. Foreign current situation and development trend of unmanned combat aircraft [C]. 2014 China's unmanned aerial vehicle (uav), 2014, 49-56.

[2]. Tang KS, Man KF, Kwong S, He Q. Genetic algorithms and their applications[J]. IEEE Signal Processing Magazine, 1996, 13: 22-37.

[3]. Kennedy J, Eberhart R. Particle swarm optimization[C]. IEEE international conference on neural networks, 1995: 1942-1949.

[4]. D Karaboga, B Basturk. A powerful and efficient algorithm for numerical function optimization: artificial bee colony (ABC) algorithm[J]. Journal of Global Optimization, 2007, 39(3): 459-471.

[5]. Au F T K,Cheng Y S,Tham L G, et al. Robust design of structures using convex models[J]. Computers and Structures, 2003, 81(28): 2611-2619.

[6]. Chen Shyi-Ming. Fuzzy backward reasoning using fuzzy Petri nets[J]. IEEE Transaction on System man and Cybernetics-part B, 2000, 30(6):846-856.

[7]. Rong Y, Wing S L, Pheng A H, et al. Improved algorithm on rule-based reasoning systems modeled by fuzzy Petri nets[C]IEEE NNS. 2002 IEEE International Conference on Fuzzy Systems: FUZZ-ZEEE'02. Honolulu: Institute of Electrical and Electronics Engineers Inc, 2002: 1204-1209.

[8]. MAWei-jiang,YAOPei-yang,ZHOUXiang-xiang. Improved method of situation nassessment in BVR aircombat[J]. Computer Engineeringand Design, 2011. 32(06): 9-13.

[9]. ZHANGHong-bo,LIGuo-ying,DINGQuan-xin. Research on Situation Assessment in BVR Air Combat [J]. Electronics Optics \& Control, 2010. 17(04):2096-2099.

[10]. NIULv-wei, GAOXiao-guang, ZHANG Kun, making decisions on proper cooperation tactics for multiple fighters to combat from BVR [J]. Journal of Northwestern Polytechnical University, 2011, 9(6):971-977. 